\title{
Experimental model of cutaneous radiation injury in rabbits ${ }^{1}$
}

\author{
Rafael Panisi de Campos Meirelles ${ }^{\mathrm{I}}$, Bernardo Hochman ${ }^{\mathrm{II}}$, Americo Helene Junior ${ }^{\mathrm{III}}$, Rute Lellis ${ }^{\mathrm{IV}}$, Murillo Francisco Pires \\ Fragav $^{v}$, Lydia Masako Ferreira ${ }^{\mathrm{VI}}$
}

IFellow Master degree, Postgraduate Program of Translational Surgery, Federal University of Sao Paulo/Paulista School of Medicine (UNIFESP/ EPM), Sao Paulo-SP, Brazil. Resident at Faculty of Medical Sciences of Santa Casa of Sao Paulo. Conception, design, intellectual and scientific content of the study, acquisition of data, surgical procedures, manuscript writing.

IIPhD, Associate Professor, Division of Plastic Surgery, Department of Surgery, UNIFESP, Sao Paulo-SP, Brazil. Conception of the study, manuscript writing, critical revision.

IIIPhD, Associate Professor, Division of Plastic Surgery, Department of Surgery, Faculty of Medical Sciences of Santa Casa of Sao Paulo, Brazil. Manuscript writing, critical revision.

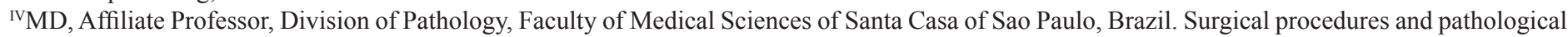
analysis.

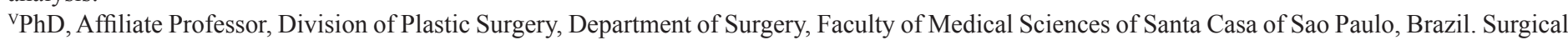
procedures, manuscript writing, critical revision.

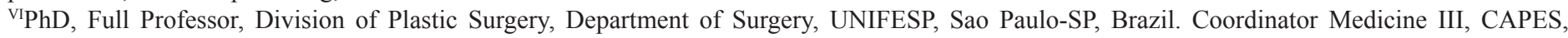
Researcher CNPq. Manuscript writing, critical revision.

\begin{abstract}
PURPOSE: To describe an experimental model of cutaneous radiation injury in rabbits.

METHODS: On this study eight six-month-old New Zealand male rabbits, with an average weight of $2.5 \mathrm{~kg}$ were used. They were distributed in four groups ( $\mathrm{n}=2$ per group). The control group did not receive radiotherapy and the others received one radiotherapy session of 2000, 3000 and 4500 cGy, respectively. Photographic analysis and histopathological evaluation of the irradiated areas were carried out.

RESULTS: After 30 days, the animals from the control group had all their hair grown. In spite of that, the animals from group 2000 cGy had a 60-day alopecia and from group 3000 cGy, a 90-day alopecia. After the 30th day, the 3000cGy group demonstrated 90-day cutaneous radiation injuries, graded 3 and 4 . One of the animals from group 4500 cGy died on the 7th day with visceral necrosis. The other from the same group had total skin necrosis. A progressive reduction of glands and blood vessels count and an increase on collagen deposition was observed.
\end{abstract}

CONCLUSION: The proposed experimental model is reproductable. This study suggests that the dosage 4500cGy is excessive and the $3000 \mathrm{cGy}$ is the most effective for this experimental model of cutaneous radiation injury in rabbits.

Key words: Radiation Injuries. Radiotherapy Dosage. Models, Animal. Rabbits. 


\section{Introduction}

Around $60 \%$ of cancer patients receive radiotherapy during their treatment ${ }^{1,2}$. The most common complication of radiotherapy is the cutaneous radiation injury. The lack of experimental models of cutaneous radiation injury is one of the research challenges ${ }^{3}$. There are some experimental models described for small animals (murine, rats and mice) ${ }^{3}$. In spite of that, there are no reproductable models in literature for medium size animals.

The rabbits, medium size animals, because of their immunological similarity to humans are the most frequently used for cutaneous tests such as the Draize test ${ }^{4}$. Their antibody development is considered resistant to radiation, differently than rats, mice and murines that aren't capable of presenting a similar immune response ${ }^{5}$.

A experimental model is a materialization of a reality and it is as similar as possible to the desired study ${ }^{6-8}$. Therefore, the development of an efficacy treatment for radiodermitis requires a reproductable experimental model with animals immunologically similar to humans, such as rabbits.

\section{Methods}

All the procedures followed the protocol approved at the Ethical Committee: UNIFESP/EPM-0264/12.

Eight six-month-old New Zealand male rabbits, with an average weight of $2.5 \mathrm{~kg}$, were used. They were distributed in four groups ( $\mathrm{n}=2$ per group). The control group did not receive radiotherapy and the others received one radiotherapy session of 2000, 3000 and $4500 \mathrm{cGy}$.

The animals were anesthetized with Ketamine intramuscular (gluteal region) and the dose was $0.5 \mathrm{ml} / \mathrm{kg}$.

The dorsal skin was epilated $(10 \times 10 \mathrm{~cm})$ with an OSTER A5 hair removal -blade size $40-10 \mathrm{~mm}$. The borders of epilation were standardized (from the occipital region, a 10x10 cm square was drawn, having the vertebral column as the mid line).

The dorsal skin was elongated and fixed between two wood sticks. The sticks were fixed with an elastic string, giving five laps on each side (Figure 1).

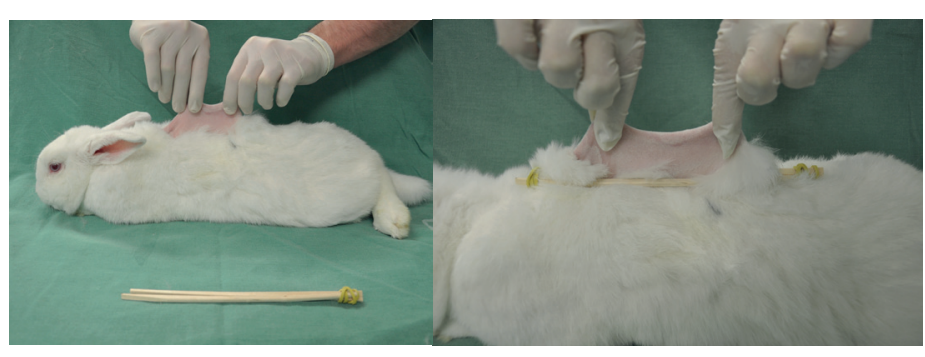

FIGURE 1 - Standardized epilation and fixation. Use of two wood sticks and elastic string with five laps on each side to elongate the dorsal skin.

The radiotherapy was performed in pairs of animals. After anesthesia, with the elongated dorsal skin, they were positioned on lateral decubitus and fixed together with three nylon 4.0 sutures, with a $10 \mathrm{~cm}$ distance between them. They were then fixed to the table with adhesive tape (Figure 2).

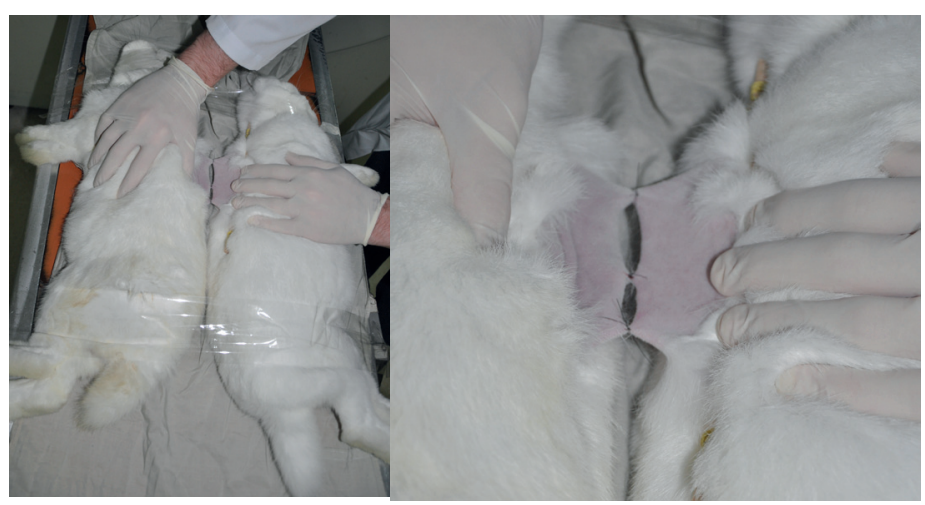

FIGURE 2 - Position: lateral decubitus of the rabbits fixed to the table with adhesive tape: $10 \mathrm{~cm}$ distance between them, fixed with nylon sutures.

The radiation machine used was ALCYON II for Telecobaltotheraphy. The distance applied was $70 \mathrm{~cm}$ and the Bolus used was $0.5 \mathrm{~cm}$. The radiation field was $5 \times 10 \mathrm{~cm}$ (width $\mathrm{x}$ length) and the dosages were 2000cGY, 3000cGY and 4500cGy.

The procedures were performed by the same researcher, assisted by a veterinarian and a radiotherapist at Arnaldo's Institute at Faculty of Medical Sciences of Santa Casa of Sao Paulo.

Serial photography of the dorsal skin surface was performed to evaluate for macroscopic evidence of radiation injury on days 15, 30, 60 and 90 post radiotherapy. The images were analyzed and classified by the same blinded radiotherapist.

The radiodermitis is classified based on skin appearance ${ }^{9-12}$. Cox et al. ${ }^{13}$ published the Acute Radiation Morbidity Scoring Criteria to classify the effects of radiotherapy, grading 0 (no reaction), 1 (light eritema, dry desquamation, epilation), 2 (moderate eritema, exudative dermatitis and moderate edema), 3 (exudative dermatitis, intense edema) and 4 (ulceration, necrosis).

The camera used was a NIKON D90 on macro and 
automatic feature, with a $30 \mathrm{~cm}$ distance from the animal.

The dorsal skin was divided in four quadrants and skin biopsy specimens were taken from the zone of irradiation on day 15, 30, 60 and 90 after external beam radiation therapy and fixed in $10 \%$ formalin ( $n=2$ per group). Specimens were paraffin-embedded and sectioned for hematoxylin and eosin staining. The histopathological analysis was performed by the same blinded pathologist. Fibrosis was assessed by picrosirius red stain (collagen content). The 'point-counting' technique proposed by Gundersen et al. ${ }^{14}$ was used to quantify the number of blood vessels, sweat glands, sebaceous glands, hair follicles and collagen using a reticulum of 100 points and 50 lines. Five random and non-coinciding fields were examined, totalling 500 points per slide at a magnification of $\mathrm{x} 200$. Points that were not incident upon solid areas were not considered. The values were expressed in percentage of positive points, in the total of points in solid areas, according to the following formula: $\mathrm{P}$ points $=(\mathrm{Pi}$ $\mathrm{x} 100) / \mathrm{P}$ parenchyma; where $\mathrm{P}$ points is the corrected percentage of marked points, $\mathrm{Pi}$ is the number of points that are incident upon the positivity of the items measured and $\mathrm{P}$ parenchyma is the total number of points that are incident upon the solid area.

\section{Results}

After 30 days of radiotherapy, the control group animals had all their hair grown. In spite of that, the animals from group 2000 cGy had a 60-day alopecia and from group 3000 cGy, a 90-day alopecia. The irradiated area from group 3000cGy demonstrated radiodermitis graded 3 (exudative dermatitis, edema) and 4 (ulceration - Figure 3) at 60 day and at 30 day, respectively. One of the animals from group $4500 \mathrm{cGy}$ died on the 7 th day after radiotherapy, because of visceral necrosis. The other animal from the same group had total skin necrosis after 30 days of radiotherapy and was sacrificed.

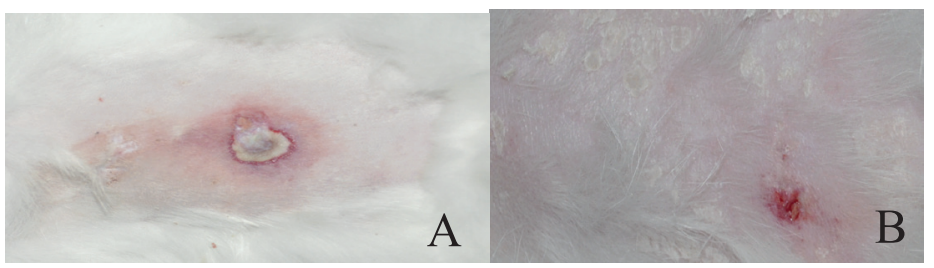

FIGURE 3 - Group 3000 cGy 30 days (A) and 60 days (B) of radiotherapy. Observe lesion graded 4 with ulceration and graded 3 with exudative dermatitis, intense edema.
The epilated area was classified based on the Oncology Radiotherapy Group Scale (grade 0-4: Figure 4) ${ }^{15}$.

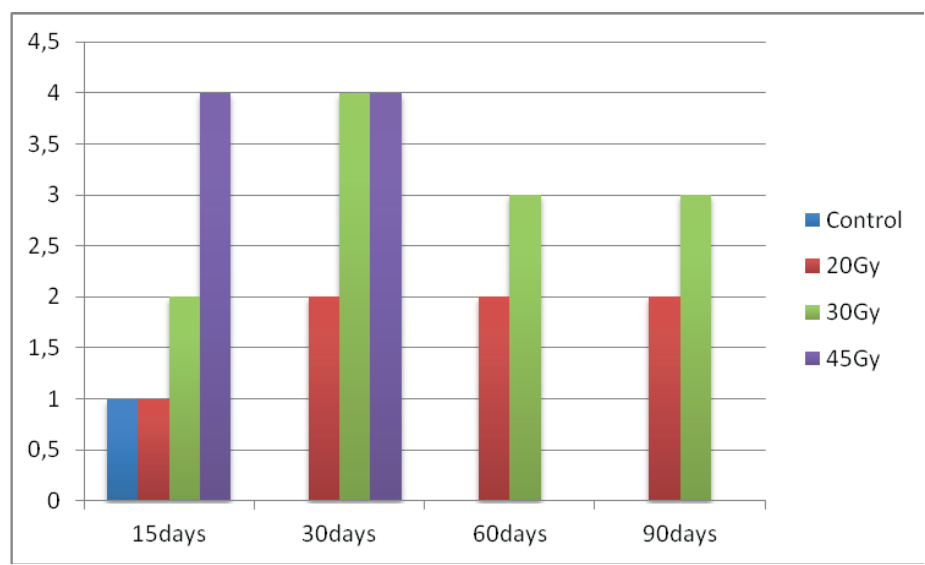

FIGURE 4 - Classification of radiodermitis ${ }^{15}$. Graded 0-4. Group 3000cGy demonstrated stable classification graded 3-4.

Rabbits exposed to radiation doses greater than 2000 cGy developed progressive skin thickening. The skin was thickened and inelastic with areas of non-healing ulceration (Figure 3). The histopathological analysis demonstrated thickening of the dermis, with exuberant and progressive collagen deposition over time (Figure 5).

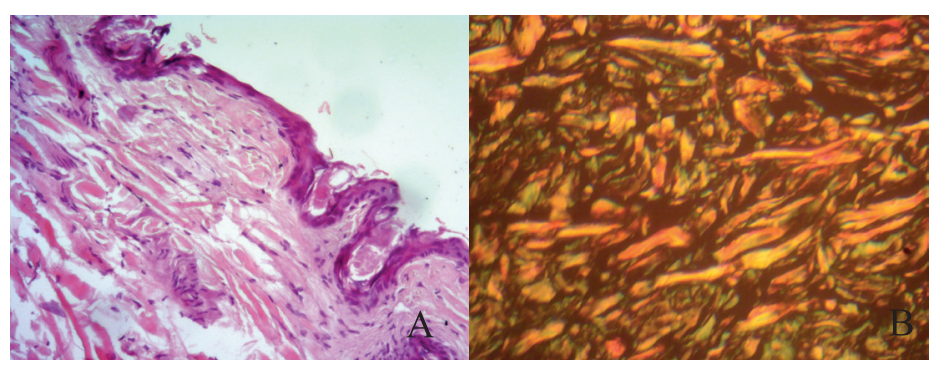

FIGURE 5 - Group 3000 cGy after 60 days of radiotherapy (Hematoxilineosin-HE-A)/(Picrosirius Red-B). A decrease on number of blood vessels, sweat glands, sebaceous glands, hair follicles is observed (A) and an increased picrosirius red staining, a marker of fibrosis (B).

A progressive reduction of glands and blood vessels count and an increase on collagen deposition was observed (Table $1)$. 
TABLE 1 - Analysis of blood vessels, glands (sebaceous, sweat glands and hair follicles) and collagen. P POINT= Pi x100/ $\mathrm{P}$ parenchyma (average results).

\begin{tabular}{|c|c|c|c|c|c|c|c|c|c|c|c|c|}
\hline & $\begin{array}{c}15 \\
\text { days }\end{array}$ & & & $\begin{array}{c}30 \\
\text { days }\end{array}$ & & & $\begin{array}{c}60 \\
\text { days }\end{array}$ & & & $\begin{array}{c}90 \\
\text { days }\end{array}$ & & \\
\hline & $\begin{array}{l}\text { Blood } \\
\text { vessels }\end{array}$ & Glands & Collagen & $\begin{array}{c}\text { Blood } \\
\text { vessels }\end{array}$ & Glands & Collagen & $\begin{array}{c}\text { Blood } \\
\text { vessels }\end{array}$ & Glands & Collagen & $\begin{array}{c}\text { Blood } \\
\text { vessels }\end{array}$ & Glands & Collagen \\
\hline Control & 2.1 & 12.4 & 10.6 & 2.4 & 14.9 & 11.2 & 2.3 & 21.7 & 11 & 2.4 & 19.2 & 11.2 \\
\hline $20 \mathrm{~Gy}$ & 1.9 & 11.2 & 16.2 & 1.6 & 7.4 & 16.4 & 1.8 & 9.1 & 18.2 & 1.6 & 8.4 & 18.4 \\
\hline 30Gy & 1.9 & 10.8 & 16.2 & 1.4 & 6.4 & 18.2 & 1.2 & 5.3 & 18.4 & 1.2 & 5.1 & 18.6 \\
\hline 45Gy & 1.8 & 10.7 & 16.4 & - & - & - & - & - & - & - & - & - \\
\hline
\end{tabular}

\section{Discussion}

Although new techniques and sources of radiotherapy have been developed, the cutaneous tolerance is frequently a limiting factor during the radiotherapy treatment ${ }^{3}$. The skin exposition to ionized radiation causes acute and chronic effects. During the acute phase, the inflammatory cells, such as macrophages, are triggered and eritema, exudative dermatitis and ulceration may appear. The chronic phase is known as a proliferative phase, with an increase of myofibroblasts. The combination of perivascular fibrosis is due to myofibroblasts activation and its effects to the endotelial cells may cause hypovascularization and chronic fibrosis ${ }^{16-20}$. This fibrosis is characterized by excessive collagen deposition, with stromal encasement of blood vessels, loss of hair follicles, and dermal thickening ${ }^{21,22}$. The progressive collagen deposition, skin thickening and alopecia were features also found in our study and mimic a human radiation injury.

The dosages used on this study were similar to the murine model described by Thanik et al. ${ }^{3}$ On their study, from the 25 treated animals, three in the highest dose groups $(\geq 4500$ cGy) had inadequate lead shielding, resulting in tail exposure with subsequent necrosis and infection, and died within the first week. On our study, both animals from group 4500 cGy did not have a desired outcome.

The alopecia was noticed on groups 2000 cGy and 3000 cGy. The alopecia noticed after radiotherapy is caused by the damage to follicular cells associated with fibrosis ${ }^{23}$. After radiotherapy, the tissue may become thick, inelastic, hyperchromic and hairless. Effects are thought to be dose, technique, and location dependent ${ }^{24}$. Our results demonstrate this dose dependency since the alopecia was more persistent on group 3000cGy.

This model accurately reproduces the changes seen in humans subjects from radiotherapy-induced injury. It was possible to observe and classify the irradiated area by photographic analysis. This classification was not previously described for an experimental study. In spite of that, it could be used, since the dosages were comparable to dose rates to human treatment protocols and the macroscopic changes were similar ${ }^{25}$.

The establishment of a reliable rabbit model to mimic the radiation-injury and study its pathogenesis and potential treatment is essential. Although, studies on radiation injury in rabbits have been described previously, there is no established rabbit model for creating a reproducible cutaneous injury pattern. Using a rabbit model offers many advantages in the study of radiation-induced cutaneous injury. It is ease handling, low cost and the rabbit, has an adequate donor site of fatty tissue, the dorsum, differently than mice ${ }^{26}$.

\section{Conclusion}

The proposed experimental model is reproductable. This study suggests that the dosage 4500 cGy is excessive and the 3000 cGy is the most effective for this experimental model of cutaneous radiation injury in rabbits. Therefore, a study with a greater sample is required to determine the most satisfactory dosage for this model. 


\section{References}

1. Porock D, Kristjanson L. Skin reaction during radiotherapy for breast cancer: the use and impact of topical agents and dressings. Eur J Cancer Care. 1999;8 (3):143-53.

2. Campbell J, Lane C. Developing a skin-care protocol in radiotherapy. Prof Nurse. 1996;12(2):105-8

Thanik VD, Chang CC, Zoumalan RA, Lerman OZ, Allen RJ Jr, Nguyen PD, Warren SM, Coleman SR, Hazen A. A novel mouse model of cutaneous radiation injury. Plast Reconstr Surg. 2011;127(2):560-8.

Phillips L 2nd, Steinberg M, Maibach HI, Akers WA. Comparison of rabbit and human skin response to certain irritants. Toxicol Appl Pharmacol. 1972;21 (3):369-82.

3. Richter M, Abou N. Cells involved in the immune response. VII. The demonstration, using allotypic markers, of antibody formation by irradiation-resistant cells of irradiated rabbits injected with normal,

allogenic bone marrow cells and sheep erythrocytes. J Exp Med. 1969;129(6):1261-73

4. Ferreira LM, Ferreira LRK. Experimental model. Historic and conceptual revision. Acta Cir Bras. 2003;18 n.spe:1-3.

5. Ferreira LM, Hochman B, Barbosa MVJ. Experimental models in research. Acta Cir Bras. 2005;20 Suppl. 2:28-34.

6. Hochman B, Ferreira LM, Vilas Bôas FC, Mariano M. Experimental model in hamsters (Mesocricetus auratus) to study heterologous graft of scars and cutaneous deseases in plastic surgery. Acta Cir Bras. 2004;19 Suppl 1:69-78.

7. Campbell J, Lane C. Developing a skin-care protocol in radiotherapy. Prof Nurse. 1996;12(2):105-8.

8. Boots-Vickers M, Eaton K. Skin care for patients receiving radiotherapy. Prof Nurse. 1997;14(10):706-8.

9. Rice AM. An introduction to radiotherapy. Nurs Stand. 1997;12(3):49-54.

10. Naylor W. Management of acute radiotherapy induced skin reactions: a literature review. Eur J Oncol Nurs. 2001;5 (4):221-33.

11. Cox JD, Stetz J, Pajak TF. Toxicity criteria of the Radiation Therapy Oncology Group (RTOG) and the European Organization for Research and Treatment of Cancer (EORTC). Int J Radiat Oncol Biol Phys. 1995;30(3):1341-6.

12. Gundersen HJ, Bendtsen TF, Korbo L, Marcussen N, Moller A, Nielsen K. Some new, simple and efficient stereological methods and their use in pathological research and diagnosis. APMIS 1988;96:379-94

13. Hymes S, Strom E, Fife C. Radiation dermatitis: clinical presentation, pathophysiology, and treatment. J Am Acad Dermatol. 2006;54 (1):28-46.

14. Desmouliere A. Factors influencing myofibroblast differentiation during wound healing and fibrosis. Cell Biol Int. 1995;19(5):471-6.

15. Gabbiani G. Modulation of fibroblastic cytoskeletal features during wound healing and fibrosis. Pathol Res Pract. 1994;190(9-10):8513.

16. Powell DW, Mifflin RC, Valentich JD, Crowe SE, Saada JI, West AB. Myofibroblasts. I. Paracrine cells important in health and disease. Am J Physiol. 1999;277( Pt 1):C1-9.

17. Martin M, Lefaix J, Delanian S. TGF-beta1 and radiation fibrosis: a master switch and a specific therapeutic target? Int J Radiat Oncol Biol Phys. 2000;47(2):277-90.

18. Flanders KC, Sullivan CD, Fujii M. Mice lacking Smad3 are protected against cutaneous injury induced by ionizing radition. Am J Pathol. 2002;160(3):1057-68.

19. Archambeau JO, Pezner R, Wasserman T. Pathophysiology of irradiated skin and breast. Int J Radiat Oncol Biol Phys. 1995;31:1171-85.
20. O'Sullivan B, Levin W. Late radiation-related fibrosis: pathogenesis, manifestations, and current management. Semin Radiat Oncol. $2003 ; 13: 274-89$

21. Malkinson FD, Keane JT. Radiobiology of the skin: review of some effects on epidermis and hair. J Invest Dermatol. 1981;77:133-8.

22. Lawenda BD, Gagne HM, Gierga DP. Permanent alopecia after cranial irradiation: dose-response relationship. Int J Radiat Oncol Biol Phys. 2004;60:879-87.

23. Shakespeare TP, Dwyer M, Mukherjee R, Yeghiaian-Alvandi R, Gebski V. Estimating risks of radiotherapy complications as part of informed consent: the high degree of variability between radiation oncologists may be related to experience. Int J Radiat Oncol Biol Phys. 2002;54:647-53.

24. Fraga M, Helene A, Nakamura F, Lellis R, Kikuchi W, Esteban D. Comparative study of the integration and viability of autonomised and nonautonomised autologous fat tissue grafts e experimental model in rabbits. J Plast Reconstr Aesthet Surg. 2008;61:1044-8.

\section{Correspondence:}

Lydia Masako Ferreira

Universidade Federal de São Paulo

Escola Paulista de Medicina

Rua Napoleão de Barros, $715 / 4^{\circ}$ andar

04024-900 São Paulo - SP Brasil

Tel.: (55 11)55760-4118

Fax: (55 11)5571-6579

rpcmeirelles@yahoo.com.br

lydia.dcir@epm.br

Received: July 11, 2013

Review: Sept 10, 2013

Accepted: Oct 14, 2013

Conflict of interest: none

Financial source: none

${ }^{1}$ Research performed at Laboratory Animal Facility, Faculty of Medical Sciences of Santa Casa of Sao Paulo and Plastic Surgery Division, Federal University of Sao Paulo/Paulista School of Medicine (UNIFESP/ EPM), Brazil. Part of Master degree thesis, Postgraduate Program in Translational Surgery. Tutor: Lydia Masako Ferreira. 